This is an electronic reprint of the original article. This reprint may differ from the original in pagination and typographic detail.

Author(s): Forsman, Jukka T.; Seppänen, Janne-Tuomas; Mönkkönen, Mikko; Thomson, Robert L.; Kivelä, Sami M.; Krams, Indrikis; Loukola, Olli J.

Title: Is it interspecific information use or aggression between putative competitors that steers the selection of nest-site characteristics? A reply to Slagsvold and Wiebe

Year: $\quad 2018$

Version:

Please cite the original version:

Forsman, J. T., Seppänen, J.-T., Mönkkönen, M., Thomson, R. L., Kivelä, S. M., Krams, I., \& Loukola, O. J. (2018). Is it interspecific information use or aggression between putative competitors that steers the selection of nest-site characteristics? A reply to Slagsvold and Wiebe. Journal of Avian Biology, 49(3), Article jav-01558.

https://doi.org/10.1111/jav.01558

All material supplied via JYX is protected by copyright and other intellectual property rights, and duplication or sale of all or part of any of the repository collections is not permitted, except that material may be duplicated by you for your research use or educational purposes in electronic or print form. You must obtain permission for any other use. Electronic or print copies may not be offered, whether for sale or otherwise to anyone who is not an authorised user. 


\section{Is it interspecific information use or aggression between putative competitors that steers the selection of nest-site characteristics? A reply to Slagsvold and Wiebe.}

Jukka T. Forsman ${ }^{1}$, Janne-Tuomas Seppänen ${ }^{2}$, Mikko Mönkkönen ${ }^{3}$, Robert L. Thomson ${ }^{4}$, Sami M. Kivelä ${ }^{5}$, Indrikis Krams ${ }^{5,6}$ and Olli J. Loukola ${ }^{1,7}$

${ }^{1}$ Department of Ecology and Genetics, POB 8000, FI-90014 University of Oulu, Finland ${ }^{2}$ Open Science Centre, University of Jyvaskyla, PO Box 35, 40014 University of Jyvaskyla, Finland ${ }^{3}$ Department of Biological and Environmental Sciences, POB 35, FI-40014 University of Jyvaskyla, Finland

${ }^{4}$ FitzPatrick Institute of African Ornithology, DST-NRF Centre of Excellence, University of Cape Town, Rondebosch, 7701, South Africa

${ }^{5}$ Department of Zoology, Institute of Ecology and Earth Sciences, University of Tartu, Vanemuise 46, EE-51014 Tartu, Estonia

${ }^{6}$ Department of Zoology and Animal Ecology, University of Latvia, Rīga, Latvia

${ }^{7}$ Department of Biological and Experimental Psychology, School of Biological and Chemical Sciences, Queen Mary University of London, London E1 4NS, UK

Corresponding author: Jukka T. Forsman . Department of Ecology and Genetics, POB 8000, FI-90014 University of Oulu, Finland. E-mail: jukka.forsman@oulu.fi

Decision date: $27-N o v-2017$

This article has been accepted for publication and undergone full peer review but has not been through the copyediting, typesetting, pagination and proofreading process, which may lead to differences between this version and the Version of Record. Please cite this article as doi: [10.1111/jav.01558].

'This article is protected by copyright. All rights reserved.' 


\section{Abstract}

A growing number of studies have demonstrated that heterospecific individuals with overlapping resource needs - putative competitors - can provide information to each other that improves the outcomes of decisions. Our studies using cavity nesting resident tits (information provider) and migratory flycatchers (Ficedula spp., information user) have shown that Selective Interspecific Information Use (SIIU) can result in flycatchers copying and rejecting the apparent nest-site feature preferences of tits, depending on a perceivable fitness correlate (clutch size) of the tits. These, and other results on the interspecific information use, challenge the predictions of traditional theory of species coexistence. Recently, Slagsvold and Wiebe (2017) proposed an alternative hypothesis, the Owner Aggression Hypothesis $(\mathrm{OAH})$, to explain our results. Their main points of critique are: 1) a lack of evidence that flycatchers make visits into tit nests prior to nesting and 2) flycatchers do not have an ability to assess tit clutch size. According to Slagsvold and Wiebe, interspecific aggression between tits and flycatchers, not information use, is the mechanism explaining our results. In this reply we show that part of Slagsvold and Wiebe's criticism is based on mischaracterization of the assumptions of SIIU, resulting in misinterpretations of our results. We also provide new evidence that flycatchers (mostly males) frequently visit tit nests prior to settlement and can acquire information about tit clutch size and thereby on the quality of the tutoring tit individual and its decisions. In short, as intriguing as $\mathrm{OAH}$ is, we suggest that 1) some of the assumptions are highly speculative and lack evidence, while 2) our earlier experiment (Loukola et al. 2013) has clearly demonstrated the importance of the visible clutch size of tits for flycatcher decisions. Therefore, SIIU can more parsimoniously than $\mathrm{OAH}$ explain the behaviour of flycatchers.

A growing number of studies demonstrate that heterospecific individuals, even putative competitors, are used as a source of information in important decisions. The presence, density, behaviour, and fitness correlates of others are used as cues, in selecting habitat (Mönkkönen et al. 1990, Forsman et al. 1998, Fletcher 2007, Sebastián-Gonzáles et al. 2011, Samplonius and Both 2017, Szymkowiak et al. 2017) or site (Forsman et al. 2002, Seppänen and Forsman 2007, Miller et al. 2013; see also Kivelä et al. 2014) of offspring production, in offspring investment (Forsman et al. 2012), in predator recognition (Magrath et al. 2015, Emmering and Schmidt 2011, Parejo et al. 2012) and for finding food, or foraging techniques (Slagsvold and Wiebe 2011, Dawson and Chittka 2014, Farine et al. 2015). These studies highlight that interspecific information use can have significant ecological effects and, most fundamentally, challenge the predictions of traditional theories of interspecific competition and species coexistence. Resource use overlap with other species does not always result in avoidance of such species or divergence in behaviour or ecology.

Perhaps the best studied system from the point of view of heterospecific information use is the cavity nesting bird community of resident tits (e.g. Great tit, Parus major) and migratory flycatchers (Pied and Collared flycatcher, Ficedula hypoleuca and F. albicollis). We have shown that flycatchers use tit territory choices (Forsman et al. 2002) and tit density (Forsman et al. 2008) as a cue in habitat choices and in offspring investment decisions, which results in fitness benefits for flycatchers (Forsman et al. 2002, 2007). More recently, we developed an experimental design, Apparent Novel Niche Experiment (ANNE), 
with which we have examined how interspecific information is used in the selection of nest-site characteristics. ANNE uses abstract symbols (e.g., triangle or circle) that are associated with a natural niche element, such as nest or shelter site. When applied to the tit-flycatcher system, symbols are attached around the entrance hole of the nest-box occupied by a great tit, while the alternative symbol is attached on an adjacent empty nest-box 3-5 meters away (Fig. 1). Two equivalent vacant nest-boxes adjacent to each other, differing only by the type of symbol (triangle or circle) at their entrance, are offered 25-30 m from the tit nest for flycatchers to settle in (Fig. 1). This design creates apparent choice of the resident tit towards one of the symbols while flycatchers must choose between two nest-boxes differing only in the symbol associated with the box. Importantly, these unnatural symbols have no ecological relevance. This allows testing responses towards these symbols by excluding an individual's previous experience, and by isolating behavioural strategies of interest from confounding factors such as adaptive responses to naturally occurring features or events in the environment (see also Alatalo and Mappes 1996).

A series of ANNEs have shown that flycatchers not only copy the apparent nest-site feature preferences of tits (Seppänen and Forsman 2007), but that flycatchers may also selectively either copy or reject tits' symbol preferences, depending on the perceived fitness (clutch size) of the tits (Forsman and Seppänen 2011, Seppänen et al. 2011, Loukola et al. 2013). This does not mean that nest-site features need to have, either in the experiment or outside of it, any causal relationship with breeding success. Individuals with good breeding performance plausibly make better choices, meaning that copying their choices - any choices (e.g. nest site choices, foraging decisions, anti-predator strategies) - is potentially adaptive (Henrich and Gil-White 2001, Laland 2004, Galef and Laland 2005). Conversely, choices of poorly performing individuals are plausibly poor, and thus should be rejected to avoid potentially harmful outcomes. 'Copy-the-successful' and 'Reject-the-unsuccessful' strategy can lead to adopting a better than average behaviour without investing much resources in personal sampling of the environment.

Furthermore, extending this strategy to other species may give access to beneficial behaviours that otherwise would be available only by the slow process of genetic evolution - or not at all. For example migratory species cannot respond to selection pressures occurring on their breeding grounds when the species is at the wintering grounds.

Slagsvold and Wiebe (2017) propose an alternative hypothesis to explain our findings. They suggest that the choices of flycatchers are instead governed by the aggression of tits. Their Owner Aggression Hypothesis $(\mathrm{OAH})$ rests on two general ecological principles, which in themselves are plausible. First, individuals are expected to be aggressive towards other individuals, including heterospecifics, that have overlapping resource needs and which try to settle in the vicinity of their nests. Second, individuals are expected to choose breeding sites that minimize predation risk. However, the OAH makes three assumptions that are not as straightforward, and we detail those now.

First, Slagsvold and Wiebe (2017) argue that individuals should try to avoid choosing nest (or re-nesting) sites that are similar to sites where nests (their own or others) already occur. This is because the functional response and dynamic search image of predators (Martin 1996, 1998) may lead to higher

'This article is protected by copyright. All rights reserved.' 
predation risk in those types of microhabitats. This assumption may only be half the ecological story, however, because the absence of other breeding attempts in a microhabitat might just result from responses to higher predation risk, or indeed from realization of predation risk at those sites; dead men (or birds) tell no tales.

Second, Slagsvold and Wiebe (2017) argue that tits not only defend extra cavities (vacant nest boxes) in their territory, but that i) in vacant cavities well away from their own nest, the tits defend more aggressively the nest site that is different from the cavity hosting their current nest, and ii) their aggressiveness increases with clutch size. In this scenario, differences in aggression over nest site types would result from the first assumption above (preference for nesting site differing from own current nest). The correlation between aggression and clutch size would result from the phenotype of the tit (e.g. size, condition, personality) and/or increasing aggressiveness when breeding advances from egg-laying to incubation. While personality of great tits affect their nest defense behaviour (Vrublevska et al. 2014), it is not clear whether the above assumptions are plausible.

Third, the OAH hypothesis implicitly assumes that great tits consistently use a strategy where they aggressively defend vacant nests-sites that include an opposite nest-site characteristics compared to the characteristics of their current, still successful, nesting attempt. Natural selection indeed can favour such a bet-hedging strategy where risk of nesting failure is decreased by re-nesting behaviour (i.e., all eggs are not in the same basket). However, it is unlikely that it would pay to invest time and energy in defending a resource that is not needed and has no value at that moment. If aggression is involved, it most likely stems from nest predation and adaptive behaviour to reduce nest predators' functional response to the location of birds' nests (Martin 1996, Martin \& Martin 2001a,b). However, such aggressive defense should include all possible nest-sites in the vicinity (Martin \& Martin 2001a,b), not just those with opposing characters compared to the current nesting attempt. Great tits indeed can perceive the nest-site features that are used by other co-existing species and those left unoccupied, and recollect and use that past information in own choices in the next breeding season (Forsman et al. 2014). Whether great tits proactively secure own preferences and possible needs in the future is a fascinating challenge for future studies. However, at present, this assumption is highly speculative.

Slagsvold and Wiebe (2017) label the original hypothesis of our research program the Interspecific Cue Hypothesis (ICH). However, we refer to our hypothesis here as Selective Interspecific Information Use (SIIU) instead because it better reflects the diverse utilization of interspecific information than ICH. Slagsvold and Wiebe question some alleged assumptions of SIIU, and particularly conclusions drawn from flycatcher responses to tit clutch size (Seppänen et al. 2011, Loukola et al. 2013, also Seppänen and Forsman 2007).

Part of Slagsvold and Wiebe's (2017) criticism is based on mischaracterization of assumptions of SIIU and misinterpretations of our results. Here, we will correct each of the alleged assumptions (see Table 2 in Slagsvold and Wiebe 2017), and clarify the interpretation of demonstrated flycatcher responses to tit 
clutch size, and provide supplementary data on tit breeding stage (laying vs complete clutch) on the day when the flycatcher choice was recorded. We also provide new data on the visitation rates of flycatchers in great tit nests in early spring (see below, Assumptions 3 and 4), which was one of the key points in Slagsvold and Wiebe's criticism.

\section{Alleged Assumption 1. External appearance of a tit nest provides relevant information to flycatchers}

Slagsvold and Wiebe (2017) question the possible (fitness) benefits that flycatchers can gain by copying choices of nest-site features. They wrongly argue that our hypothesis assumes that the external appearance of natural nest sites indicate beneficial features. They correctly point out that the variation in natural cavities in terms of volume, depth, moisture, ectoparasite load etc. is large, and that optimal nestsite conditions are likely to be subtly different for different species, but fail to note that these considerations are entirely irrelevant for the SIIU.

First, obviously there is no conceivable benefit of copying/rejecting choices of a novel visual nest-site feature varying only in shape (triangle or circle, Fig. 1). This is a crucially important design feature of the experiment. The experiment requires the alternative choices to be ecologically and visually neutral and evolutionarily novel, to isolate the behavior of interest (copying/rejecting) from other ecological factors, from unconditioned responses and from an individual's history and experience. By using novel neutral symbols differing only in shape, we can expect that the study animals do not have any innate or learned (via trial-and-error) preferences confounding the question of interest.

Second, and a conceptually more important point, is that SIIU does not require that flycatchers directly benefit from copying/rejecting nest site choices at all, even in natural situations. SIIU assumes that individuals derive a net benefit, over their lifetime, from the ability and propensity to copy/reject choices made by individuals of other species. Given the rapid changes and high variability in community composition and ecological dynamics, there is no reason to expect that this ability would evolve to be used only in the context of choosing nest sites, or only in relation to particular heterospecifics, or to be fine-tuned to variation in nest site features. A specific event where the copying/rejecting ability is applied can be meaningless in terms of fitness, or it can even have a fitness cost, as long as the net fitness outcome of exhibiting this behaviour is positive over an individual's lifetime. We do not know, but fully expect, that SIIU can occur throughout a flycatcher's life - for example when choosing how to forage at a migration stop-over site.

Demonstration of SIIU in a context where the choice is unlikely to have direct adaptive value gives more, not less, support for the SIIU hypothesis. The ANNE design efficiently tests whether flycatchers have an innate, generalized behavioural strategy of using the choices of local resident tits. By showing a strong association between simulated tit preference and flycatcher choices, we can make strong inferences about the existence of information use in flycatchers. All our field experiments (Seppänen and Forsman 2007, Forsman et al. 2011, Seppänen et al. 2011, Loukola et al. 2013) consistently demonstrate that flycatchers 
indeed possess the ability and propensity to respond to tit choices by copying or rejecting their apparent choices - the experiments do not assume or seek to demonstrate that this is limited to external features of nest sites or that direct fitness benefits result from nest site features. The nest sites, and the symbols, are tools - not subjects - of this research.

Nevertheless, aspects of nest sites may have direct effects of flycatcher fitness. For example, if 10 cavities in deciduous trees and 10 similar cavities in conifers are inhabited by zero and five active tit nests, respectively, then by copying the apparent conifer choice of tits, flycatchers may avoid a local predator individual that has created a search image for nests in deciduous trees. Resident tits may either avoid breeding in deciduous trees through earlier experience, or tits that had initiated nests in deciduous trees have been predated. Association between type of nest site and any fitness-relevant variable that is difficult to estimate for an arriving flycatcher (such as nest predation risk, future nest-site microclimate, incidence of disease and parasites) but which is revealed by choices or outcomes for individuals of other species, can make copying/rejecting nest-site types directly adaptive (Seppänen et al. 2007) - but this is in no way required by SIIU. Flycatchers do gain fitness benefits by choosing to breed in the tit territory (Forsman et al. 2002) and it is intriguing, yet a demanding challenge for future studies to show the underlying causal mechanism, and whether the same applies also for finer scales of nest-site choices.

\section{Alleged Assumption 2. Tit clutch size is a relevant cue}

Slagsvold and Wiebe (2017) deem that SIIU assumes that clutch size must be a relevant cue revealing information about the nest site. They correctly point out that a nest predation event usually takes away the whole clutch, and if larger clutches reflect better foraging conditions and smaller clutches reflect poorer foraging conditions, then the benefit can be gained by simply settling in or avoiding the general vicinity, no need to copy or reject nest-site type choices. But SIIU does not assume that clutch size is a relevant cue about the quality of a nest site. SIIU assumes that clutch size (or some other observable factor) is a relevant cue about the quality of the demonstrator individual.

In all our studies (Seppänen and Forsman 2007, Forsman et al. 2011, Seppänen et al. 2011, Loukola et al. 2013), we have consistently based our hypothesis on the theory of evolutionary psychology and social learning. An exhausting amount of both theoretical and empirical evidence demonstrate that tutors of high observable quality should be, and are, copied more often than poorer quality individuals (e.g., Boyd and Richerson 1985, Henrich and Gil-White 2001, Laland 2004, Galef and Laland 2005). From the flycatcher point of view, it is entirely irrelevant why a successful individual makes choices that are worth copying and why choices of poorly performing individuals should be avoided. Overall however, successful individuals are likely to make better choices in any situation and unsuccessful individuals will tend to make poor choices (Henrich and Gil-White 2001, Laland 2004, Galef and Laland 2005). Actively rejecting choices of poorly performing individuals is intriguing, and its ecological and evolutionary consequences have not been explored - to our knowledge our experiments are the first to demonstrate this in the wild. 
Recently Cole et al. (2012) provided compelling evidence why copying the choices of tits with high clutch size may be adaptive. They showed that great tit females with large clutch sizes were associated with better problem-solving skills, smaller foraging ranges and less time investment in foraging compared to females with smaller clutches. Hence, the clutch sizes of resident tits are an honest signal of behavioural repertoires and/or competency in novel decision-making. The challenge for future experiments is to create experimental designs that demonstrate whether and how SIIU operates in other contexts than nest site choice, e.g. in adopting foraging techniques, diet, or predator avoidance.

An important element in the information value of the tit clutch size for flycatchers is the timing of breeding. Most tits initiate breeding and egg-laying well before arrival of flycatchers, meaning that clutches are complete (information is honest) and visible (incubated eggs are not covered) when majority of flycatchers are choosing nest-sites. Indeed, in Seppänen et al. (2011), all tutoring great tits in Latvia and in central Finland (Jyväskylä; except 1 pair out of 57) and the majority (84.4\%) of tits in northern Finland (Oulu) were incubating at the time flycatchers made their symbol choice.

\section{Assumptions 3 and 4. Inspection of tit nests and its costs.}

SIIU assumes that flycatchers inspect tit nests prior to choosing a nest-site and assess the current number of eggs or the final clutch size in tit nests. Slagsvold and Wiebe (2017) argue that i) there is little empirical evidence showing prospecting of tit nests and ii) entering tit nests occurs primarily when there is shortage of potential nest-sites (Slagvold 1978) and the function of this behaviour is to claim the cavity, not to assess the number of tit eggs (Slagsvold and Wiebe 2017).

We acknowledge that flycatcher visits to tit nests is imperative for the SIIU of which our publications have not previously provided much direct evidence. Here, we provide new data showing visitation rates of pied flycatcher to great tit nests.

We monitored 82 active great tit nests by filming each nest for 48 hours during the tits' egg laying (67 nests) or incubation period (15 nests) in Oulu in 2011 (35 nests) and 2012 (47 nests) using miniature video surveillance cameras (model: CCD Bird Box Camera 1/4" SONY 420tvl with 11 LED's) with infrared motion detectors that were attached inside the nest box roof. These great tit nests were not manipulated and most recordings were done when tits' had 4-5 eggs. Prior to recording, we attached dummy cameras in the nest boxes to allow tits to habituate to the presence of the foreign object in their nest box. From the recorded videos, we measured the frequency of flycatcher visits into the tit nest or, if possible, at the entrance of the box, how much time they spent in the nest and, if possible, what was the sex of the visiting flycatcher.

Of the 67 nests which were filmed during the egg laying stage, 28 (41.8\%) were visited by pied flycatchers (similar in both years; $40.9 \%$ and $42.2 \%$ ). In most visits (24), flycatchers entered the tit nest. Single male visits were clearly most frequent ( 21 events) followed by male and female visits ( 6 events) and only one single female visit. The average number of flycatcher visits was 10.8 (range 1 - 93 visits) 
during the $48 \mathrm{~h}$ filming period in the great tit nests visited at least once. Average visit duration was 156 seconds (range $5-816 \mathrm{~s}$ ). Only one of the 15 great tit nests filmed during the incubation period was visited by a (male) pied flycatcher.

Visits to the great tit nests cause mortality among pied flycatchers. We have found 16 dead flycatchers (one observation with two dead males in the same box) in great tit nest boxes $(9.7 \%$, dead flycatcher in 15 boxes of 154) in Finland during the years 2011 - 2013. Of these 12 were males. In Latvia, flycatcher mortality due to tits seems to be slightly lower, plausibly due to higher asynchrony in breeding. Among 370 nest boxes between 2010-2015, 12 dead male flycatchers and 2 females (3.78\%) have been found (I. Krams, unpublished data). All dead flycatchers were found in tit nests with incomplete clutches.

To conclude, flycatchers frequently visit great tit nests and most visits are made solely by males.

Visitation rates are highest during the egg-laying period when tit parents do not spend much time in the nest or in the vicinity of the nest. Most flycatcher deaths also occurred during the egg-laying period. These results demonstrate that flycatchers visit tit nests even though vacant nests sites are available. We agree with Slagsvold and Wiebe that it is possible that flycatchers sometimes enter tit nests to claim ownership or check availability but it does not exclude the possibility that they may simultaneously assess clutch size.

\section{Assumption 5. Flycatchers ability to assess clutch size.}

Slagsvold and Wiebe (2017) argue that it is unlikely that flycatchers can assess the number of tit eggs. Their main arguments are as follows. First, the sex-ratio of dead flycatchers found in tit nests is male biased (roughly 70-80\%; Merilä and Wiggins 1995, Ahola et al. 2007) and males predominantly enter tit nests. Because males lack a brood patch, tactile stimulation (using the brood patch) is claimed to be an unlikely mechanism to assess egg number. Second, even if flycatchers are capable of visual qualitative or quantitative discrimination of eggs (of which no evidence exists), cavities are too dark to allow visual assessment. Third, acquiring a benchmark to which low or high clutch size can be determined is argued to be difficult and dangerous, and benchmarks might be highly variable depending on local conditions.

Our new data suggests that $75 \%$ of the observed visits to tit nests were done by males. In addition, $75 \%$ the dead flycatchers found in tit nests were males; in line with the earlier findings (Merilä and Wiggins 1995, Ahola et al. 2007). How then are flycatchers able to assess tit clutch size and base their decisions on that if predominantly only males visit tit boxes, which have no proven capacity for numerical estimation of eggs and are not assumed to have a major role in nest-site choice decisions?

We suggest plausible mechanisms of assessment, which we acknowledge, requires further study. First, while the capacity for numerical estimation is most common in brood parasites (Haywood 2016), there is evidence for numerical ability in other birds too (Hunt et al. 2008, Garland and Low 2014), even in this specific context of assessing clutch size to choose nest site (Odell and Eadie 2010). Male flycatchers may have capability to visually estimate egg number based on the size of the contrasting light area of pale eggs

'This article is protected by copyright. All rights reserved.' 
against dark nest linings or using tactile stimulation. Second, recent evidence suggests that also males have independent effects on reproductive strategies (Brommer and Rattiste 2008, Germain et al. 2016), including nest-site choice decisions (Loukola et al. 2012, Jaakkonen et al. 2015; see also Kivelä et al. 2014). Our recent study indeed suggested that in the collared flycatcher, pairs with a young male have a higher probability to copy simulated tit symbol preference than pairs with an old male irrespective of the female's age (Morinay, J., Forsman, J.T, Kivelä, S.M., Gustafsson, L., Doligez, B., unpublished manuscript). Hence, flycatcher males most likely have a stronger effect on the choice of the nest-sites than previously thought. In addition, the observed pattern of rejecting and copying of tit symbols is reinforced by those females that visit in tit nests. Flycatcher females have a proven capacity to assess at least the difference between 4 vs 13 eggs because dummy tit nests with these numbers of plastic eggs impacted their investment in offspring in terms of clutch size and egg mass (Forsman et al. 2012). Interestingly, recently Samplonius and Both (2017) showed that experimentally advanced and delayed breeding phenology of tits affected the habitat choices of female flycatchers while males seemed to ignore this cue. Even though Samplonius and Both (2017) could not isolate the mechanism how females assess the breeding state of the tits, it nevertheless suggests that they possess such capabilities.

Darkness of the cavities may hamper visibility. However, cavities need not to be well lit for assessing egg number, as birds are clearly able to e.g. pick up and remove broken egg shells and nestling feces which are smaller items than a clutch of eggs. Moreover, many birds (Stevens and Cuthill 2007), including flycatchers (Siitari et al. 2002), have a capacity to perceive ultraviolet light and it is possible that UVreflecting eggs stand out brightly against less reflecting nest lining. The eggs of the cavity nesting species indeed have a higher UV-reflectance than those of non-cavity nesting species, which is suggested to be adaptive because it enhances parents' ability to detect eggs in dark conditions (Avilés et al. 2006).

It remains speculative what benchmark flycatchers use to discern low vs high clutch size, and how that benchmark is acquired (discussed in Seppänen et al. 2011). Various environmental conditions, such as quality and quantity of food (e.g., McCleery et al. 2004, Charmantier et al. 2006) or nest predation risk (e.g., Julliard et al. 1997, Zanette et al. 2011) can on average decrease or increase the standard deviation of the local average clutch size by about $5-25 \%$, or by $0.4-1.5$ eggs. Such relatively low variation around the mean in full clutches is perhaps not that difficult to control for by visiting a few nests prior to decision-making. Flycatchers may also have a genetically determined or learned benchmark to which the observed quantity of tit eggs is compared. Stronger responses of older female flycatchers to the number of dummy tit eggs compared to first time breeders (Forsman et al. 2012) suggests that previous experience about own or others' clutches may enhance ability to judge the size of observed clutch sizes.

\section{Assumption 6. Aggression between tits and flycatchers}

Overlap in resource use between species that results in costs is expected to select for adaptations that reduce the costs of coexistence. One such adaptation is aggression between species, which indeed can reduce the spatial overlap in species occurrence and, hence, decrease the related costs (Martin and Martin 2001 a,b). Slagsvold and Wiebe (2017) argue that an important untested prerequisite of the SIIU is that 
tits do not interfere with flycatchers' nest-site selection and, in particular, do not try to prevent flycatchers nesting in a box and symbol that they want to preserve for own later use in case of nesting failure.

We have indeed assumed that flycatchers are free to settle in either of the vacant 'flycatcher boxes'. We think this assumption is robust for two main reasons. First, we have never observed aggression between the species nor found dead flycatchers in our 'flycatcher boxes' situated $25-30 \mathrm{~m}$ from the tit nests. If tits aggressively prevent flycatchers from nesting in these vacant nest boxes, we should frequently find dead flycatchers in them. Second, we have shown experimentally that great tits suffer, while flycatchers benefit, from coexistence (Forsman et al. 2002, 2007). In addition, the nest cavity density (four/ 1ha) created by our experimental designs (e.g., Seppänen et al. 2011) falls within the range of the natural density of cavities (Remm et al. 2006) suggesting that we have not enforced birds to breed unnaturally close to each other. Rather than being aggressive towards flycatchers, we have shown that great tits cover their eggs under experimentally simulated presence of flycatchers (Loukola et al. 2014). This piece of evidence also implies that the number of visible eggs is important for both great tits and flycatchers.

\section{Selective Interspecific Information Use hypothesis vs. the Owner Aggression Hypothesis}

As intriguing as Slagsvold and Wiebe's (2017) hypothesis is, several lines of reasoning suggest that, without any further evidence for the assumptions of the OAH (see above), it is not a more plausible hypothesis than the SIIU for four reasons (see also Samplonius 2017):

1. If the intention of great tits is to prevent flycatchers to breed in their territory or in the sought after nest site (box), there is no reason to expect great tits to wait until the start of incubation until they increase their aggression. Tit males devote part of their time to mate-guarding but females have no such restrictions, in particularly during the egg laying period. As shown by Martin and Martin (2001 a,b), if the more aggressive species suffers from the co-occurrence of other species, behaving aggressively throughout the settlement phase and displacing heterospecifics from the shared habitat altogether is adaptive. Moreover, if great tits increase their aggression after the clutch is complete, flycatchers breeding near the tits should strongly shift from non-matching to matching symbol choices compared with flycatchers breeding near the tits that are still laying eggs. However, copying clearly increased linearly as a function of increasing clutch size of tits in all three spatially distant populations studied (Seppänen et al. 2011).

2. Overlap in nest-site feature preferences are not actively avoided in a conspecific context. If great tits aim to choose the safest nest-site feature possible (Slagsvold and Wiebe 2017), they would be expected to also avoid overlap in nest-site feature choices with conspecifics to reduce the risk of nest predation. However, in a symbol experiment with dummy tit nests with high (13 eggs) and low (4 eggs) numbers of plastic eggs, we showed that, while simulated clutch size did not affect symbol choices of great tits, the age of the males had an effect (Loukola et al. 2012). Pairs with old males strongly copied the apparent choice of earlier settled conspecifics, while pairs with young males tended to reject the choice. Jaakkonen et al. (2015) also showed a similar effect of male age in a field experiment with real tit tutors and nests.

'This article is protected by copyright. All rights reserved.' 
3. Our new (Assumption 3 and 4) and previous data (Assumption 5, Merilä and Wiggins 1995, Ahola et al. 2007), clearly demonstrate that both male and female flycatchers frequently visit great tit nests in the beginning of the nesting period. Visits are male dominated, and males likely have a larger role in the selection of nest-sites than previously assumed (Loukola et al. 2012, Kivelä et al. 2014, Morinay et al. unpublished manuscript). This provides a plausible mechanism for how flycatcher male dominated nest visitation rates and current egg number in tit nests can be transmitted into copying or rejection choice of the flycatcher pair.

4. Experimental manipulation of the clutch size of great tits by Loukola et al. (2013) provides conclusive support for the SIIU. We manipulated great tit clutches to be either large (13 eggs) or small (5 eggs) during the whole arrival period of flycatchers. We also monitored whether eggs (information) were covered or not because there is variation among tit females how well they cover eggs prior to incubation (Haftorn and Slagsvold 1995, pers. obs.). Our results showed that flycatchers reject and copy simulated tit preferences when tit clutch size was small or large, respectively, but only when the eggs were not covered. If eggs were covered and the clutch size information was not available, flycatchers made opposite choices compared to when the eggs were fully visible. This pattern was especially clear in the large clutch size treatment (Loukola et al. 2013). One could speculate that clutch size manipulation may have affected the behavior of tit parents (cf. Slagsvold and Wiebe 2017). However, the visibility of the egg number information totally changed the response of flycatchers, and conclusively demonstrated the effect and importance of the clutch size as such in affecting flycatcher choices.

\section{Conclusions and questions for future studies}

We have provided new evidence and arguments that provide support for the existence of interspecific information use in birds. Most importantly, because 1) both male and female flycatchers frequently visit tit nests in early spring, 2) males plausibly affect nest-site choice (Loukola et al. 2012, Kivelä et al. 2014), and 3) the strong effect of the visibility of tit eggs on flycatcher choice (Loukola et al. 2013), we argue that SIIU is more likely than Slagsvold and Wiebe's (2017) OAH. To test both hypotheses simultaneously, an experiment with a similar design as in Seppänen et al. (2011) and Loukola et al. (2013) could be used. Tit eggs could then be covered completely with animal hair during egg-laying period, and flycatcher symbol choices monitored. If flycatchers' rejection-copying behaviour is similar to previous studies, then SIIU is falsified. If choices are random or rejection dominated (cf. Loukola et al. 2013), then OAH is falsified.

Many unknowns persist about mechanisms of information acquisition and possible co-evolution between species engaged in 'information source and information user' interactions. Aggressive interactions between species using similar resources are common (e.g. Martin and Martin 2001 a,b), including between tits and flycatchers (Slagsvold 1975, this study). Developing Slagsvold and Wiebe's (2017) OAH hypothesis further, and by taking variation among individuals into account in the general level of aggressiveness and nest defence behaviour (Verbeek et al. 1996, Dingemanse et al. 2002, Hollander et al. 2008), it is possible that the personality of the information source may select for certain types of 
information users in their vicinity. This may shape the social environment because interactions of conand heterospecifics can have indirect genetic effects on each other and the mode of social interactions itself has been suggested to be heritable and under natural selection (Bijma et al. 2007, Wilson 2014).

Another theme calling for more research is the mechanisms of acquiring information and implementing it in decision-making.

Acknowledgements - We thank Jakub Szymkowiak for commenting the manuscript. Funding from Marie Curie Intra-European Fellowship (MEIF-CT-2003-500554), Academy of Finland (projects 122665 and 125720) and Kone Foundation (to JTF) has made many ANNE experiments and this study possible. 


\section{References}

Ahola, M. P., Laaksonen, T., Eeva, T. and Lehikoinen, E. 2007. Climate change can alter competitive relationships between resident and migratory birds. - J. Anim. Ecol. 76: 1045-1052.

Alatalo, R. V. and Mappes, J. 1996. Tracking the evolution of warning signals. - Nature 382: 708-710.

Avilés, J. M., Soler, J. J. and Péres-Contreras, T. 2006. Dark nests and egg colour in birds: a possible functional role of ultraviolet reflectance inegg detectability. Proc. R. Soc. B 273: 2821-2829.

Bijma, P., Muir, W., van Arendonk, J. A. M. 2007. Multilevel selection 1: Quantitative genetics of selection and response to selection. - Genetics 175: 277-288.

Boyd, R. and Richerson, P. J. 1985. Culture and the Evolutionary Process. - Univ. Chicago Press.

Brommer, J. E. and Rattiste, K. 2008. "Hidden" reproductive conflict between mates in a wild bird population. Evolution 62: 2326-2333.

Charmantier, A., Perrins, C. M., McCleery, R. H., and Sheldon B. C. 2006. Evolutionary response to selection on clutch size in a long-term study of the Mute Swan. - Am. Nat. 167: 453-465.

Cole, E. F., Morand-Ferron, J., Hinks, A. E. and Quinn, J. L. 2012. Cognitive Ability Influences Reproductive Life History Variation in the Wild. - Curr. Biol 22:1808-1812.

Dawson, E. H. and Chittka, L. 2012. Conspecific and heterospecific information use in bumblebees. PLoSONE 7(2): e31444. doi:10.1371./journal.pone.0031444.

Dingemanse, N. J., Both, C., Drent, P. J. van Oers, K. and van Noordwijk, A. J. 2002. Repeatability and heritability of exploratory behaviour in great tits from the wild. - Anim. Behav. 64: 929-938.

Emmering, Q. C. and Schmidt, K. A. 2011. Nesting songbirds assess spatial heterogeneity of predatory chipmunks by eavesdropping on their vocalizations. J. Anim. Ecol. 80: 1305-1312.

Farine, D. R., Aplin, L. M., Sheldon, B. C. and Hoppit, W. 2015. Interspecific social networks promote information transmission in wild songbirds. - Proc. R. Soc. B 282: 20142804.

Fletcher RJ Jr. 2007. Species interactions and population density mediate the use of social cues for habitat selection. - J. Anim. Ecol. 76: 598-606.

Forsman, J. T., Mönkkönen, M., Helle, P. and Inkeröinen, J. 1998. Heterospecific attraction and food resources in migrants' breeding patch selection in northern boreal forests. - Oecologia 115: 278-286.

Forsman, J. T., Seppänen, J.-T. and Mönkkönen M. 2002. Positive fitness consequences of interspecific interaction with a potential competitor. - Proc. R. Soc. Lond. B 269:1619-1623.

Forsman, J. T., Thomson, R.L. and Seppänen, J.-T. 2007. Mechanisms and fitness effects of interspecific information use between migrant and resident birds. - Behav. Ecol. 18: 888-894.

Forsman, J.T., Hjernquist, M.B., Taipale, J. and Gustafsson, L. 2008. Competitor density cues for habitat quality facilitating habitat selection and investment decisions. - Behav. Ecol. 19: 539-545.

Forsman, J.T. and Seppänen, J.-T. 2011 Learning what (not) to do: testing rejection and copying of simulated heterospecific behavioural traits. - Anim. Behav. 81: 879-883.

Forsman, J. T., Seppänen, J.-T. and Nykänen, I. L. 2012. Observed heterospecific clutch size can affect offspring investment decisions. - Biol Lett. 8: 341-343.

'This article is protected by copyright. All rights reserved.' 
Forsman, J. T., Kivelä, S. M., Jaakkonen, T., Seppänen, J.-T., Gustafsson, L. and Doligez, B. 2014 Avoiding perceived past resource use of potential competitors affects niche dynamics in a bird community. - BMC Evol.Biol. 14: 175.

Galef, B. G. Jr and Laland, K. N. 2005 Social learning in animals: Empirical studies and theoretical models. BioScience 55: 489-499.

Garland, A. and Low, J. 2014. Addition and subtraction in wild New Zealand robins. Behav. Processes 109. DOI: $10.1016 /$ j.beproc.2014.08.022

Germain, R. R., Wolak, M. E., Arcese, P., Losdat, S. and Reid, J. M. 2016. Direct and indirect genetic and fine-scale location effects on breeding date in song sparrows. - J. Anim. Ecol. 85: 1613-1624.

Haftorn, S. and Slagsvold, T. 1995. Egg covering in birds: description of the behaviour in tits (Parus spp.) and a test of hypotheses of its function. - Fauna norw. Ser. C, Cinclus 18: 85-106.

Haywood, S. 2016. Can birds count eggs in the nests? - Ibis 158: 195-198.

Henrich, J. and Gil-White, F. J. 2001. The evolution of prestige: Freely conferred status as a mechanism for enhancing the benefits of cultural transmission. - Evol. Hum. Behav. 22: 165-196.

Hollander, F. A., Van Overveld, T., Tokka, I. and Matthysen, E. 2008. Personality and nest defense in the Great tit (Parus major). - Ethology 114: 405-412.

Hunt, S., Low, J. and Burns, K. C. 2008. Adaptive numerical competence in food-hoarding songbird. Proc. R. Soc. B 275: 2373-2379.

Jaakkonen, T., Kivelä, S. M., Meier, C. M. and Forsman, J. T. 2015. The use and relative importance of intraspecific and interspecific social information in a bird community. - Behav. Ecol. 26: 55-64.

Julliard, R., McCleery, R.H., Clobert, J. and Perrins, C.M. 1997. Phenotypic adjustment of clutch size due to nest predation in the great tit. - Ecology 78: 394-404.

Kivelä, S. M., Seppänen, J.-T., Ovaskainen, O., Doligez, B., Gustafsson, L., Mönkkönen, M. and Forsman, J. T. 2014. The past and the present in decision-making: the use of conspecific and heterospecific cues in nest site selection. - Ecology 95: 3428-3439.

Laland, K. N. 2004. Social learning strategies. - Learn. Behav. 32: 4-14.

Loukola, O. J., Seppänen, J.-T. and Forsman, J. T. 2012. Intraspecific social information use in the selection of nest site characteristics. Anim. Behav. 83:629-633.

Loukola, O. J., Seppänen, J.-T., Krams, I., Torvinen, S. S. and Forsman, J. T. 2013. Observed fitness may affect niche overlap in competing species via selective social information use. - Am. Nat. 182: 474483.

Loukola, O. J, Laaksonen, T., Seppänen, J.-T. and Forsman, J. T. 2014. Active hiding of social information from information parasites. - BMC Evol Biol 14:32. doi:10.1186/1471-2148-14-32

Magrath, R. D., Haff, T. M., Fallow, P. M. and Radford, A. N. 2015 Eavesdropping on heterospecific alarm calls: from mechanisms to consequences. - Biol. Rev. 90: 560-586.

Martin, P. R. and Martin, T E. 2001a. Ecological and fitness consequences of species coexistence: a removal experiment with wood warblers. - Ecology 82: 189-206.

Martin, P. R. and Martin, T. E. 2001b. Behavioral interactions between coexisting species: song playback experiments with wood warblers. - Ecology 82: 207-218.

'This article is protected by copyright. All rights reserved.' 
Martin, T. E. 1996. Fitness costs of resource overlap among coexisting bird species. - Nature 380: $338-$ 340 .

Martin T. E. 1998. Are microhabitat preferences of coexisting species under selection and adaptive? Ecology 79: 656-670.

McCleery, R. H., Pettifor, R. A., Armbruster, P., Meyer, K., Sheldon B. C. and Perrins C. M. 2004. Components of variance underlying fitness in a natural population of the Great tit Parus major. Am. Nat. 164: E62-E72.

Merilä, J. and Wiggins, D. A. 1995 Interspecific competition for nest holes causes adult mortality in the collared flycatcher. - Condor 97:445-450.

Miller, C. W., Fletcher, R. J. Jr. and Gillespie, S. R. 2013. Conspecific and heterospecific cues override resource quality to influence offspring production. - PlosOne 8: e70268.

Mönkkönen, M., Helle, P. and Soppela, K. 1990. Numerical and behavioural responses of migrant passerines to experimental manipulation of resident tits (Parus spp.): Heterospecific attraction in northern breeding bird communities? - Oecologia 85: 218-225.

Odell N. S. and Eadie J. M. 2010. Do wood ducks use the quantity of eggs in a nest as a cue to the nest's value? Behav. Ecol. 21, 794-801.

Parejo, D., Avilés, J. M. and Rodríguez, J. 2012 Alarm calls modulate the spatial structure of a breeding owl community. - Proc. R. Soc. B. 279: 2135-2141.

Remm, J., Lõhmus, A. and Remm, K. 2006. Tree cavities in riverine forests: What determines their occurrence and use by hole-nesting passerines? - Forest Ecol. Manag. 221: 267-277.

Samplonius, J. M. 2017. Does territory owner aggression offer an alternative explanation to patterns in heterospecific information use studies? A comment on Slagsvold \& Wiebe. - J. Avian Biol. DOI: 10.1111/jav.01567.

Samplonius, J. M. and Both, C. 2017. Competitor phenology as a social cue in breeding site selection. - J. Anim. Ecol. 86: 615-623.

Sebastián-Gonzáles, E., Sánchez-Zapata, J. A, Botella, F. and Ovaskainen, O. 2010. Testing the heterospecific attraction hypothesis with time-series data on species co-occurrence. - Proc. R. Soc. B 277: 2983-2990.

Seppänen, J.-T. and Forsman, J. T. 2007. Interspecific social learning: novel preference can be acquired from a competing species. - Curr. Biol. 17: 1-5.

Seppänen, J.-T., Forsman, J. T., Mönkkönen, M. and Thomson, R. L. 2007. Social information use is a process across time, space, and ecology, reaching heterospecifics. - Ecology 88: 1622-1633.

Seppänen, J.-T., Forsman, J. T., Mönkkönen, M., Krams, I. and Salmi, T. 2011. New behavioural trait adopted or rejected by observing heterospecific tutor fitness. - Proc. R. Soc. Lond. B 278: 17361741.

Siitari, H., Honkavaara, J., Huhta, E. and Viitala, J. 2002. Ultraviolet reflection and female mate choice in the pied flycatcher, Ficedula hypoleuca. - Anim. Behav. 63:97-102.

Slagsvold, T. 1975. Competition between the great tit Parus major and the pied flycatcher Ficedula hypoleuca in the breeding season. - Ornis Scand. 6: 179-190.

'This article is protected by copyright. All rights reserved.' 
Slagsvold, T. and Wiebe, K. L. 2011. Social learning in birds and its role in shaping a foraging niche. Phil. Trans. R Soc. B 366: 969-977.

Slagsvold, T. and K. L. Wiebe. 2017. On the use of heterospecific information for nest site selection in birds. - J. Avian Biol. 48: 1-6.

Stevens, M. and Cuthill, I. C. 2007. Hidden messages: are ultraviolet signals a special channel in avian communication. - BioScience 57: 501-507.

Szymkowiak, J., Thomson, R. L. and Kuczynski, L. 2017. Interspecific social information use in habitat selection decisions among migrant songbirds. - Behav. Ecol. 28. doi:10.1093/beheco/arx029

Verbeek, M. E. M., Boon, A. and Drent, P. J. 1996. Exploration, aggressive behaviour and dominance behaviour if pairwise confrontations of juvenile male great tits. - Behaviour 133: 945-963.

Vrublevska, J., Krama, T., Rantala, M.J., Mierauskas, P., Todd M. Freeberg, T.M. and Krams, I.A. 2014. Personality and density affect nest defence and nest survival in the great tit. - Acta Ethologica 18: 111-120.

Wilson, A. J. 2014. Competition as a source of constraint on life history evolution in natural populations. - Heredity 112: 70-78.

Zanette, L. Y, White, A. F, Allen, M. C. and Clinchy, M. 2011 Perceived predation risk reduces the number of offspring songbirds produce per year. - Science 334: 1398-1401.

'This article is protected by copyright. All rights reserved.' 


\section{FIGURE LEGEND}

Figure 1. Schematic figure about the experimental design of the Apparent Novel Niche Experiment (ANNE) used in many studies that have examined the interspecific information use between resident tits (information source, sitting on top of the nest box) and migratory flycatchers (information user, flying bird).

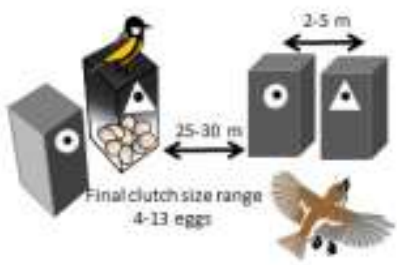

